\title{
Extraction of the Generalized Parton Distribution $H(\xi, \xi, t)$ from DVCS
}

\author{
$\underline{\text { V.A. Korotkov }}^{\mathrm{a}}$, W.-D. Nowak ${ }^{\mathrm{b}}$ \\ aIHEP, Protvino, RU-142281, Russia

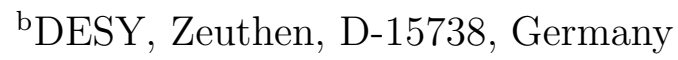

A simple way to extract the Generalized Parton Distribution $H(\xi, \xi, t)$ at small $t$ from the single beam-spin asymmetry in Deeply Virtual Compton Scattering is proposed. Projections are given for future measurements at the HERMES experiment upgraded with a recoil detector.

\section{Introduction}

First results on measurements of the single beam-spin asymmetry in Deeply Virtual Compton Scattering (DVCS) have recently been published by the HERMES [1] and CLAS [2] collaborations. Both collaborations will upgrade their apparatus to measure the DVCS process with improved accuracy.

There are four different types of twist-2 quark Generalized Parton Distributions (GPDs) contributing to the DVCS process. In the unpolarized distributions, $H^{q}(x, \xi, t)$ and $E^{q}(x, \xi, t)$, the quark helicities are summed over. The polarized distributions, $\widetilde{H}^{q}(x, \xi, t)$ and $\widetilde{E}^{q}(x, \xi, t)$, are responsible for the differences between right- and left-handed quarks. The GPDs depend on two longitudinal momentum fraction variables $x$ and $\xi$ (skewedness) and the squared momentum transfer $t$ between initial and final nucleon states. The crosssection of the DVCS process and its interference with the Bethe-Heitler $(\mathrm{BH})$ process has been considered in a number of papers [3 6]. The detailed form of the Compton amplitude can be found in Ref. [7]. The process amplitude $\mathcal{T}$ is the sum of the DVCS and BH amplitudes, $\mathcal{T}_{\mathrm{DVCS}}$ and $\mathcal{T}_{\mathrm{BH}}$. Their interference opens the possibility to access the DVCS amplitudes [5] through a measurement of the interference term $\mathcal{I}=\mathcal{T}_{\mathrm{DVCS}} \mathcal{T}_{\mathrm{BH}}^{*}+\mathcal{T}_{\mathrm{DVCS}}^{*} \mathcal{T}_{\mathrm{BH}}$. Explicit expressions for the amplitudes of the DVCS, Bethe-Heitler and interference terms including the first subleading correction in $1 / Q$ have been calculated in Ref. [6] for different kinds of polarized and unpolarized initial particles. The four-fold cross-section for the process $e(k) p\left(P_{1}\right) \rightarrow e^{\prime}\left(k^{\prime}\right) p^{\prime}\left(P_{2}\right) \gamma\left(q_{2}\right)$ with an unpolarized proton target depends on the Bjorken variable $x_{\mathrm{B}}$, the lepton energy fraction $y=P_{1} \cdot q_{1} / P_{1} \cdot k, t=\left(P_{2}-P_{1}\right)^{2}$, and the azimuthal angle $\phi$ between the lepton and hadron scattering planes:

$\frac{d \sigma}{d x_{\mathrm{B}} d y d|t| d \phi}=\frac{\alpha^{3} x_{\mathrm{B}} y}{8 \pi Q^{2} \sqrt{1+4 x_{\mathrm{B}}^{2} M^{2} / Q^{2}}}\left|\frac{\mathcal{T}}{e^{3}}\right|^{2}$.

Here, $Q^{2}=-q_{1}^{2}$ and $q_{1}=k-k^{\prime}$. The squared amplitudes $\left|\mathcal{T}_{\mathrm{BH}}\right|^{2}$ and $\left|\mathcal{T}_{\mathrm{DVCS}}\right|^{2}$, and the interference term $\mathcal{I}$ are expressed in terms of finite sums of Fourier harmonics with respect 
to the azimuthal angle $\phi$ (see Eq.s (25-27) in Ref. [6]). Note that for the DVCS process the skewedness variable can be determined by $\xi=x_{\mathrm{B}} /\left(2-x_{\mathrm{B}}\right)$.

\section{Projection for a future DVCS measurement at HERMES}

The HERMES collaboration will upgrade their apparatus with a recoil detector [8] to measure the single beam-spin and the beam-charge asymmetries using an unpolarized proton target. Prospects for a measurement of the single beam-spin asymmetry are investigated in this paper. For the projection of the expected statistical accuracy the theoretical approach of Ref. [6] was adopted. In the analysis described below five different versions of parameterizations for twist-2 GPDs are used. They are referred to as (A) to (E) (for details see Ref. [9]). The twist-3 GPDs were taken to be zero for simplicity.

The projections of the statistical accuracy for measurements of the single beam-spin asymmetry at HERMES were calculated for an integrated luminosity of $2 \mathrm{fb}^{-1}$, corresponding to one nominal year of data taking. The HERMES acceptance for the detection of the scattered electron, the real gamma and the recoil proton has been taken into account. The following kinematic cuts were applied: $E_{e^{\prime}}>3.5 \mathrm{GeV}, E_{\gamma}>0.8 \mathrm{GeV}$, $P_{p^{\prime}}>0.2 \mathrm{GeV} / \mathrm{c}, W^{2}>4 \mathrm{GeV}^{2}, Q^{2}>1 \mathrm{GeV}^{2}$, and $15<\Theta_{\gamma \gamma^{*}}<70 \mathrm{mrad}$. Here, $W^{2}=\left(P_{2}+q_{2}\right)^{2}$ and $\Theta_{\gamma \gamma^{*}}$ is the polar angle between virtual and real photons.

The remarkably improved accuracy in measuring the variable $t$ with the recoil detector will allow accurate studies of kinematic dependences of the asymmetry. For this purpose it is convenient to define an appropriate $\sin \phi$ moment' of the asymmetry:

$$
A_{L U}^{\sin \phi}=2 \cdot \frac{\int_{0}^{2 \pi} d \phi \sin \phi\{d \sigma(\vec{e} p) / d \phi-d \sigma(\overleftarrow{e} p) / d \phi\}}{\int_{0}^{2 \pi} d \phi\left\{d \sigma\left(\overrightarrow{e^{\top}} p\right) / d \phi+d \sigma(\overleftarrow{e} p) / d \phi\right\}}
$$

The projections for the statistical accuracy of the moment $A_{L U}^{\sin \phi}$ as a function of $x_{\mathrm{B}}$ for two regions in $t$ are shown in Fig. 1.

\section{Extraction of the Generalized Parton Distribution $H(\xi, \xi, t)$}

The asymmetry moment depends on all four DVCS amplitudes, $\mathcal{H}, \widetilde{\mathcal{H}}, \mathcal{E}, \widetilde{\mathcal{E}}$, which embed the four Generalized Parton Distributions $H^{q}(x, \xi, t), E^{q}(x, \xi, t), \widetilde{H}^{q}(x, \xi, t), \widetilde{E}^{q}(x, \xi, t)$ (see e.g. Ref. 9]). A general method for a simultaneous extraction of all four GPDs from the asymmetry moments has not been worked out, yet. As will be shown in the following, information on the imaginary part of the DVCS amplitude $\mathcal{H}, \operatorname{Im} \mathcal{H}$, can nevertheless

be obtained under quite reasonable assumptions from future measurements of the $A_{L U}^{\sin \phi}$ moment at HERMES, as outlined above. The main part of the DVCS cross section contributing to the denominator of Eq. (2) is proportional to

$$
\begin{aligned}
\mathcal{C}_{\text {unp }}^{\text {DVCS }}=\frac{1}{\left(2-x_{\mathrm{B}}\right)^{2}}\{ & 4\left(1-x_{\mathrm{B}}\right)\left(\mathcal{H} \mathcal{H}^{*}+\widetilde{\mathcal{H}} \widetilde{\mathcal{H}}^{*}\right)-x_{\mathrm{B}}^{2}\left(\mathcal{H} \mathcal{E}^{*}+\mathcal{E} \mathcal{H}^{*}+\widetilde{\mathcal{H}} \widetilde{\mathcal{E}}^{*}+\widetilde{\mathcal{E}} \widetilde{\mathcal{H}}^{*}\right) \\
& \left.-\left(x_{\mathrm{B}}^{2}+\left(2-x_{\mathrm{B}}\right)^{2} \frac{t}{4 M^{2}}\right) \mathcal{E} \mathcal{E}^{*}-x_{\mathrm{B}}^{2} \frac{t}{4 M^{2}} \widetilde{\mathcal{E}} \widetilde{\mathcal{E}}^{*}\right\}
\end{aligned}
$$

\footnotetext{
${ }^{1}$ Note that a factor 2 is used in Eq. (2) to obey to the HERMES definition of the moments [1]
} 


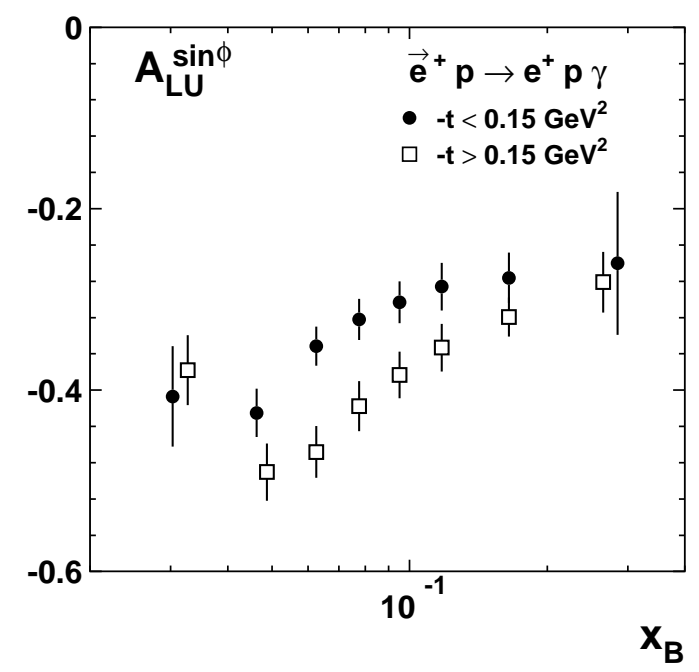

Figure 1. Projected statistical accuracy for a future HERMES measurement of the $A_{L U}^{\sin \phi}$ moment of the DVCS single beam-spin asymmetry as a function of $x_{\mathrm{B}}$ for two regoins in -t. The calculations, using GPD model version (E) (see Ref. [9]), are based on an integrated luminosity of $2 \mathrm{fb}^{-1}$.

The numerator of Eq. (2) is proportional to $\operatorname{Im} \mathcal{C}_{\text {unp }}^{\mathcal{I}}$, where

$\mathcal{C}_{\text {unp }}^{\mathcal{I}}=F_{1} \mathcal{H}+\frac{x_{\mathrm{B}}}{2-x_{\mathrm{B}}}\left(F_{1}+F_{2}\right) \widetilde{\mathcal{H}}-\frac{t}{4 M^{2}} F_{2} \mathcal{E}$

Here, $F_{1}$ and $F_{2}$ are the Dirac and Pauli form factors. From the expressions (3) and (4) it may be seen that at small values of $t$ and $x_{\mathrm{B}}$ the main contributions come from the first terms of both expressions. This observation can be checked numerically using different models for GPDs. In the following the region of small $-t\left(<0.15 \mathrm{GeV}^{2}\right)$ is considered.

Expression (3) can be regrouped into two terms, $\mathcal{C}_{\text {unp }}^{\text {DVCS }}=B_{1}+B_{2}$, with

$B_{1}=4 \frac{1-x_{\mathrm{B}}}{\left(2-x_{\mathrm{B}}\right)^{2}}(\operatorname{Im} \mathcal{H})^{2}$

and $B_{2}$ representing the remainder of $\mathcal{C}_{\text {unp }}^{\text {DVCS }}$. The relative contributions of the $\mathrm{BH}$ and DVCS cross sections to the denominator of Eq. (2) are shown in Fig. 2. The same figure shows the relative contributions coming from the DVCS cross section parts proportional to $B_{1}$ and $B_{2}$. From the figure it may be concluded that in the kinematics considered in this paper the main component of the DVCS cross section contributing to the denominator of Eq. (2) is due to $\operatorname{Im} \mathcal{H}$. In Fig. 3 are shown the relative contributions of the three terms from Eq. (雨), induced to the numerator of Eq. (2) due to the amplitudes $\mathcal{H}, \widetilde{\mathcal{H}}$ and $\mathcal{E}$. Again, it may be concluded that the main contribution comes from $\operatorname{Im} \mathcal{H}$.

These numerical studies show that the most significant contribution to $A_{L U}^{\sin \phi}$ at small $-t\left(<0.15 \mathrm{GeV}^{2}\right)$ is originating from $\operatorname{Im} \mathcal{H}$. The sum of all remaining contributions amounts only to a few percent. This conclusion has been checked for all five GPD model versions considered in Ref. [9]. Therefore, the assumption, that the $A_{L U}^{\sin \phi}$ moment may 

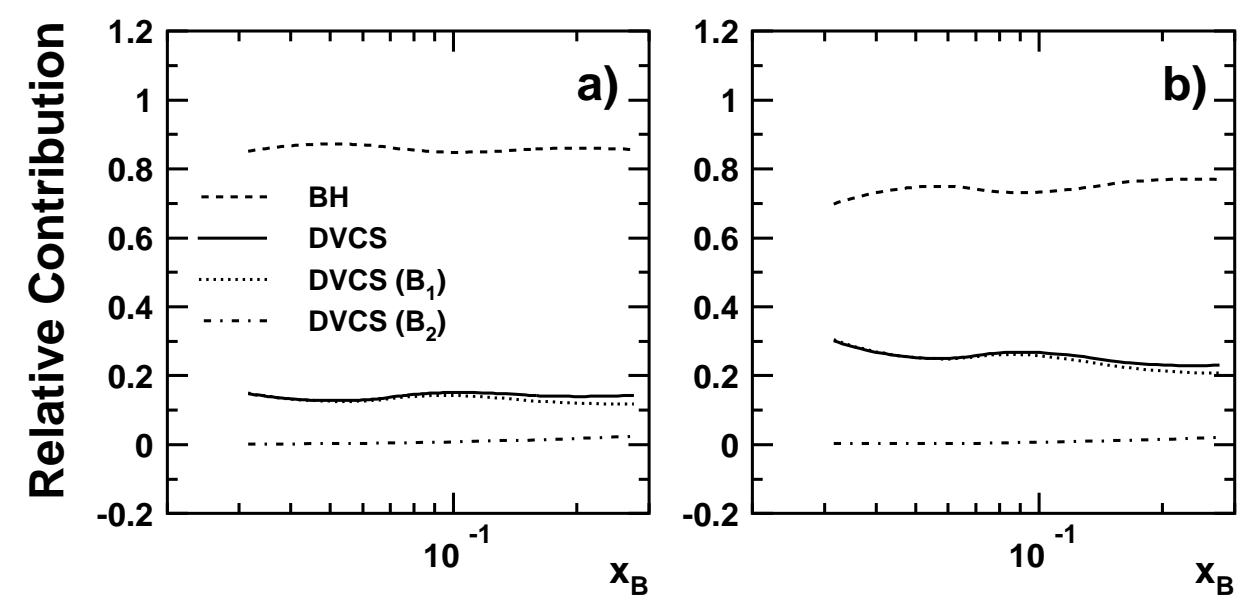

Figure 2. Relative contribution of the BH and DVCS cross sections to the denominator of $E q$. (国). The relative contributions coming from the DVCS cross section parts proportional to $B_{1}$ and $B_{2}$ are shown also. Results for the GPD versions $(A)$ and $(B)$ are shown in panels a) and b), respectively.
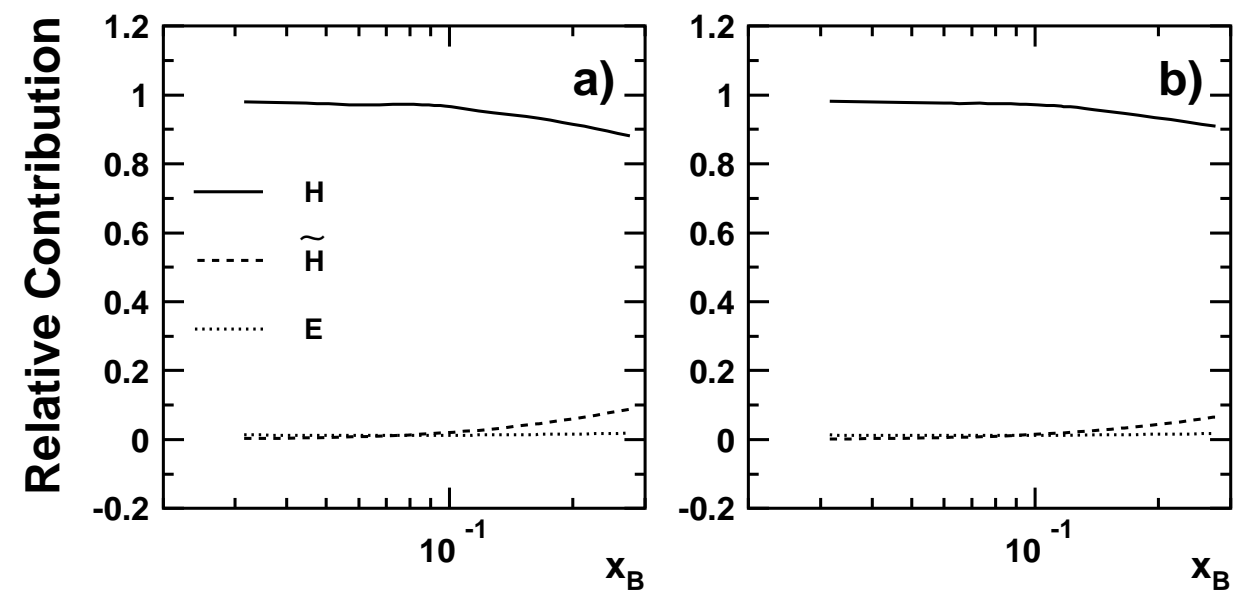

Figure 3. Relative contribution of terms from Eq. (4) induced to the numerator of Eq. (园) due to the amplitudes $\mathcal{H}, \widetilde{\mathcal{H}}$, and $\mathcal{E}$. Results for $G P D$ versions $(A)$ and $(B)$ are shown in panels a) and b), respectively.

be analysed as a function of $\operatorname{Im} \mathcal{H}$ only, appears to be quite reasonable. In this scenario, the moment (2) can be expressed in the following form:

$A_{L U}^{\sin \phi}=2 \cdot \frac{\int d \Omega \sin \phi d \sigma_{0}^{I} / d \Omega \cdot \operatorname{Im} \mathcal{H}}{\int d \Omega d \sigma^{B H} / d \Omega+\int d \Omega d \sigma_{0}^{D V C S} / d \Omega \cdot(\operatorname{Im\mathcal {H}})^{2}}$.

Here, $\Omega$ denotes all relevant variables, $d \sigma^{B H} / d \Omega$ is the cross section of the $\mathrm{BH}$ process, $d \sigma_{0}^{D V C S} / d \Omega$ is that of the unpolarized DVCS process, and $d \sigma_{0}^{I} / d \Omega$ is that of the interference term. Both $d \sigma_{0}^{I} / d \Omega$ and $d \sigma_{0}^{D V C S} / d \Omega$ are calculated assuming $\operatorname{Im\mathcal {H}}=1, \operatorname{Re} \mathcal{H}=$ $0, \widetilde{\mathcal{H}}=0, \mathcal{E}=0, \widetilde{\mathcal{E}}=0$. Based on this assumption a procedure has been developed to 
extract $\operatorname{Im} \mathcal{H}$ in a parameterized form from future HERMES measurements. A simple parameterization with two free parameters $c_{1}$ and $c_{2}$,

$\operatorname{Im} \mathcal{H}(\xi)=c_{1} \xi^{c_{2}}$

was adopted following an observation made in Ref. [10]. Fits to the projected moment asymmetry in the region $-t<0.15 \mathrm{GeV}^{2}$, performed on the basis of Eq.s (6) and (7), show good descriptions of the $x_{\mathrm{B}}$-dependence of $A_{L U}^{\sin \phi}$. The results of the fits to the projected moments for GPD model versions (A) and (B), taken as examples, are shown in Fig. 4 . Typical values of $\chi^{2} / n d f$ are $(4 \div 6) / 6$ if every point is varied in limits of $\pm 3 \sigma$ of its projected error. Deviations of the extracted function $\operatorname{Im} \mathcal{H}$ from that used as input for the projections are found to be small within the expected statistical accuracy.
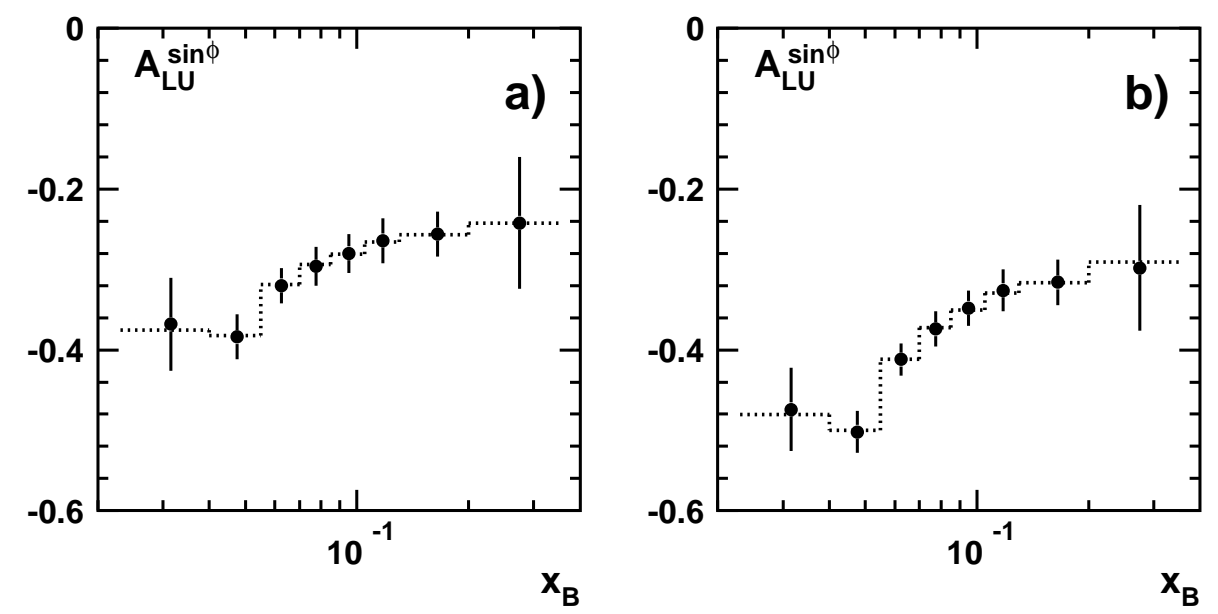

Figure 4. Results of a fit to the projected moments for GPD model versions (A) and (B) on the basis of Eq.s (6) and (19), calculated for $-t<0.15 \mathrm{GeV}^{2}$.

To leading order in $\alpha_{s}$, the determination of $\operatorname{Im} \mathcal{H}$ corresponds to a measurement of the quantity $\sum_{q} e_{q}^{2}\left(H^{q}(\xi, \xi, t)-H^{q}(-\xi, \xi, t)\right)$, taken along the diagonals $x= \pm \xi$ (cf. e.g. Eq. (19) in Ref. [9]). The fully correlated $1 \sigma$ error bands for a future measurement of this quantity at HERMES in the region $-t<0.15 \mathrm{GeV}^{2}$, as obtained after the fit, are shown in Fig. 5 for GPD model versions (A) and (B). The systematic uncertainty of this simple analysis method, as depicted in Fig. 5, was estimated to be the average of the deviations between reconstructed and input functions for all versions of GPDs and was found to be smaller than the envisaged statistical uncertainty.

\section{Conclusions}

Numerical studies carried out for different models of GPDs have shown that the most significant contribution to $A_{L U}^{\sin \phi}$ at small $-t$ and $0.03<x_{B}<0.3$ is originating from $\operatorname{Im} \mathcal{H}$. This observation allows to develop a procedure for the extraction of the Generalized Parton Distribution $H$ from future HERMES measurements of the single beam-spin asymmetry. A simple parameterization of $\operatorname{Im} \mathcal{H}$ with two free parameters allows to describe a dependence of $A_{L U}^{\sin \phi}$ on $x_{\mathrm{B}}$ at small $t$. Deviations of the extracted function 
$\operatorname{Im} \mathcal{H}$ from that used as input for the projections are small inside the expected statistical accuracy.

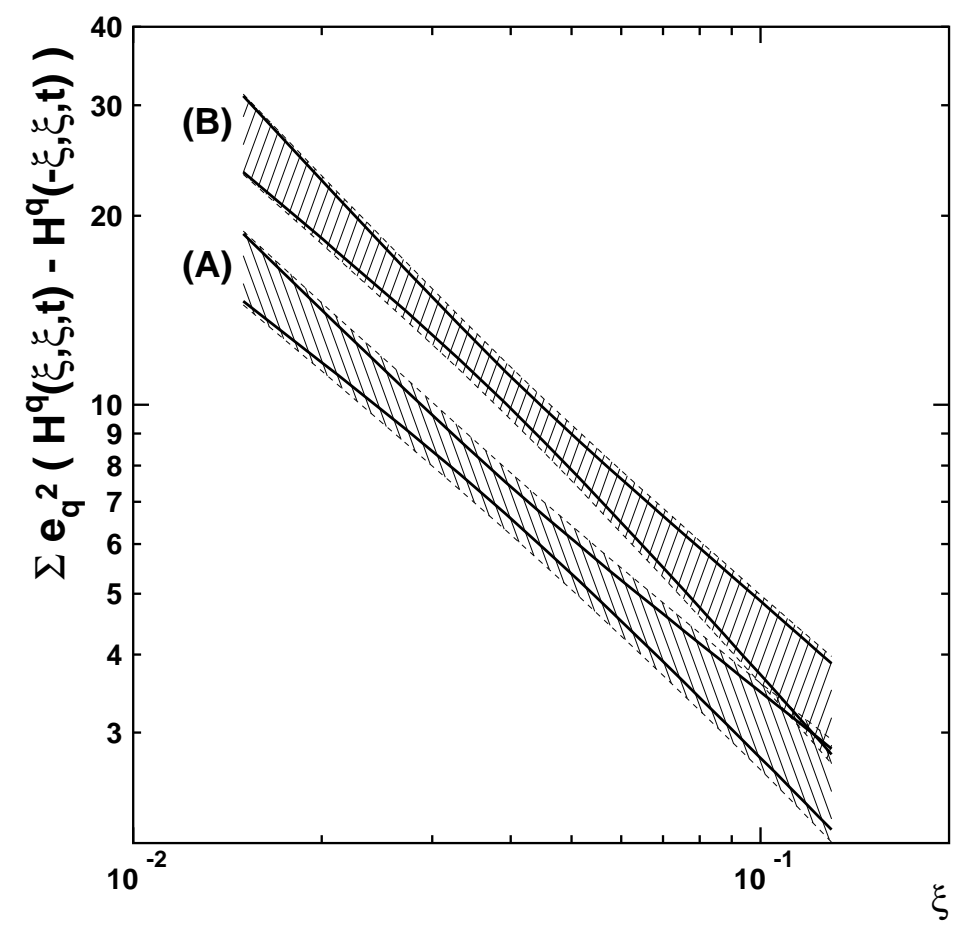

Figure 5. Projection for a future HERMES measurement, based on $2 \mathrm{fb}^{-1}$, of $\sum_{q} e_{q}^{2}\left(H^{q}(\xi, \xi, t)-H^{q}(-\xi, \xi, t)\right)$, shown in dependence of $\xi$ and $x_{\mathrm{B}}$ simultaneously. The fully correlated $1 \sigma$ error bands (solid lines) show the projected statistical accuracy for the two GPD model versions (A) and (B), chosen as examples (see Ref. [9]). The hatched bands represent the linear sum of the statistical $1 \sigma$ error and a systematic error estimated to be due to the approximations inherent in the used analysis method.

We are grateful to M. Diehl and R. Kaiser for a careful reading of the manuscript.

\section{REFERENCES}

1. A. Airapetian et al., Phys. Rev. Lett. 87 (2001) 182001.

2. S. Stepanyanian et al., Phys. Rev. Lett. 87 (2001) 182002.

3. X. Ji, Phys.Rev. D55 (1997) 7114.

4. A.V. Radyushkin, Phys.Rev. D56 (1997) 5524.

5. M. Diehl et al., Phys.Lett. B411 (1997) 193.

6. A.V. Belitsky , D. Müller, A. Kirchner, Nucl. Phys. B629 (2002) 323.

7. J. Blümlein and D. Robaschik, Nucl.Phys. B581 (2000) 449.

8. HERMES Coll., DESY PRC 02-01, 2002.

9. V.A. Korotkov and W.-D. Nowak, Eur. Phys. J. C23 (2002) 455.

10. A. Freund and M. McDermott, Phys. Rev. D65 (2002) 074008. 